\title{
Towards Detecting Need for Empathetic Response in Motivational Interviewing
}

\author{
Zixiu $\mathrm{Wu}$ \\ zixiu.wu@philips.com \\ Philips Research \\ Eindhoven, Netherlands \\ University of Cagliari \\ Cagliari, Italy
}

\author{
Rim Helaoui \\ rim.helaoui@philips.com \\ Philips Research \\ Eindhoven, Netherlands
}

\author{
Vivek Kumar* \\ Diego Reforgiato Recupero \\ Daniele Riboni \\ vivek.kumar@unica.it \\ diego.reforgiato@unica.it \\ riboni@unica.it \\ University of Cagliari \\ Cagliari, Italy
}

\begin{abstract}
Empathetic response from the therapist is key to the success of clinical psychotherapy, especially motivational interviewing. Previous work on computational modelling of empathy in motivational interviewing has focused on offline, session-level assessment of therapist empathy, where empathy captures all efforts that the therapist makes to understand the client's perspective and convey that understanding to the client. In this position paper, we propose a novel task of turn-level detection of client need for empathy. Concretely, we propose to leverage pre-trained language models and empathy-related general conversation corpora in a unique labellerdetector framework, where the labeller automatically annotates a motivational interviewing conversation corpus with empathy labels to train the detector that determines the need for therapist empathy. We also lay out our strategies of extending the detector with additional-input and multi-task setups to improve its detection and explainability.
\end{abstract}

\section{CCS CONCEPTS}

- Computing methodologies $\rightarrow$ Discourse, dialogue and pragmatics; Transfer learning; • Applied computing $\rightarrow$ Psychology.

\section{KEYWORDS}

motivational interviewing, empathy, classification, deep learning

\section{ACM Reference Format:}

Zixiu Wu, Rim Helaoui, Vivek Kumar, Diego Reforgiato Recupero, and Daniele Riboni. 2020. Towards Detecting Need for Empathetic Response in Motivational Interviewing. In Companion Publication of the 2020 International Conference on Multimodal Interaction (ICMI '20 Companion), October 2529, 2020, Virtual event, Netherlands. ACM, New York, NY, USA, 6 pages. https://doi.org/10.1145/3395035.3425228

\footnotetext{
${ }^{*}$ Currently (September 2020) his secondment at Philips Research is in progress.

Permission to make digital or hard copies of all or part of this work for personal or classroom use is granted without fee provided that copies are not made or distributed for profit or commercial advantage and that copies bear this notice and the full citation on the first page. Copyrights for components of this work owned by others than ACM must be honored. Abstracting with credit is permitted. To copy otherwise, or republish, to post on servers or to redistribute to lists, requires prior specific permission and/or a fee. Request permissions from permissions@acm.org.

ICMI '20 Companion, October 25-29, 2020, Virtual event, Netherlands

(C) 2020 Association for Computing Machinery.

ACM ISBN 978-1-4503-8002-7/20/10 . \$15.00

https://doi.org/10.1145/3395035.3425228
}

\section{INTRODUCTION}

Empathy from the counsellor side is widely recognised as essential to building counsellor-client rapport in psychotherapy [5] ${ }^{1}$. Its importance is particularly acknowledged in motivational interviewing [17] (MI), a psychotherapeutic technique that has proved successful in helping people achieve positive behaviour change by eliciting their own motivation. It is also a key aspect of psychotherapeutic interview quality. For example, according to the most used Motivational Interviewing Coding system (MITI [18]), assessing MI integrity goes through four dimensions, namely cultivating change talk, softening sustain talk, partnership, and empathy ${ }^{2}$.

While such assessments are mostly done by experts manually, recent research has explored automatic analysis and rating of therapist empathy in MI based on text [8, 9, 35], speech [34, 38], or both [37]. Nevertheless, these studies are limited since 1) they only evaluate offline, session-level counsellor empathy, instead of offering real-time advice; 2) they nearly all rely on classical machine learning methods with heavy feature engineering, yet pre-trained language models such as BERT [3] have not been explored despite their superior results in Natural Language Understanding tasks; 3) they were conducted on undisclosed datasets of MI conversations, which makes their results difficult to verify or replicate.

To address the limitations above, we introduce a novel text-based task of detecting the need for empathy in immediate, turn-level MI counsellor response, and plan to tackle it by fine-tuning largescale pre-trained language models on publicly available free-access datasets of general and therapeutic dialogues. This new task can be highly valuable for educating inexperienced coaches and providing them with real-time guidance in their first actual sessions.

We will approach the task based on two recent empathy-related dialogue datasets: PEC [40] (Persona-based Empathetic Conversation) and HighLowRolePlayMI [20]. The former comprises general conversations from Reddit ${ }^{3}$ with each annotated as empathetic or non-empathetic at dialogue-level but not turn-level. The latter consists of role-play demonstrations of high- and low-quality MI counselling from YouTube, where each conversation (transcribed) has only session-level "high" and "low" quality labels. We make the following assumptions about these datasets:

\footnotetext{
${ }^{1}$ https://www.stephenrollnick.com/three-pieces-on-empathy/

${ }^{2}$ https://casaa.unm.edu/download/miti4_2.pdf

${ }^{3}$ Reddit (https://www.reddit.com/) is an online platform consisting of a number of subforums, a.k.a. subreddits, each corresponding to a specific topic for Reddit users to discuss.
} 
(1) In the high-quality conversations in HighLowRolePlayMI (aliased as HighRolePlayMI), the therapist only shows empathy when the client needs it.

(2) Responses from the listener are predominantly empathetic in the empathetic PEC conversations and non-empathetic in the non-empathetic ones.

(3) How the skilled therapist in HighRolePlayMI decides whether to show empathy to the client based on the conversation flow is different from the manner in which the ordinary Reddit listener in the empathetic PEC conversations makes this decision before reacting to the speaker.

(4) How the therapist shows empathy HighRolePlayMI is similar to the way the listener shows empathy in the empathetic $P E C$ conversations, meaning that the empathetic responses in two different domains (therapeutic and general conversations) share similar patterns.

Assumption (1) is highly reliable because of the importance of therapist empathy in high-quality MI counselling. Assumption (2) is verified statistically by Zhong et al. [40] with "substantial" inter-annotator agreement. Assumption (3) is also very likely to be true since skilled therapists have been specially trained to engage their clients with empathy, whereas ordinary Reddit users have not. Assumption (4) is reasonable but yet to be substantiated, and we therefore will explore its reliability in our experiments. Enabled by these assumptions, our approach consists of two steps: empathy labelling of HighRolePlayMI exploiting PEC and needfor-empathy detection using HighRolePlayMI.

For empathy labelling, we will annotate each therapist utterance in HighRolePlayMI with a binary empathy-or-not label. Specifically, we will train a binary classifier (labeller) to distinguish between responses from the empathetic conversations and those from the non-empathetic ones in PEC, so that the resultant model is able to differentiate between empathetic and non-empathetic responses, based on assumption (2). The labeller will then label each therapist response in HighRolePlayMI as empathetic or non-empathetic, based on assumption (4). Note that due to assumptions (1) and especially (3), we cannot directly train a detector on the empathetic dialogues of PEC (general conversation domain) to accomplish the need-for-empathy detection task on HighRolePlayMI (therapeutic dialogue domain).

If the performance evaluation of the labeller on HighRolePlayMI reaches satisfactory levels (e.g. w.r.t. accuracy/F1), we will move on to the proposed need-for-empathy detection task. More concretely, we will train a detector on HighRolePlayMI which is now annotated with an empathy label for each therapist utterance. Given the conversation history where the last turn is from the client, the detector will reach a yes/no answer on whether empathy is needed in the immediate therapist response to the latest client utterance.

Both the labeller and the detector will be based on pre-trained language models and trained end-to-end. We will also experiment with additional-input regimes and multi-task designs to enhance the ability of the detector and increase its explainability.

In summary, our contributions are as follows:

- We propose a new need-for-empathy detection task, which aims to provide the human therapist with real-time advice on whether to show empathy in their response to the client.
- We propose an automatic empathy labelling method that could annotate therapist empathy, using publicly available free-access datasets of empathy-related general dialogues.

- We present our plan for approaching the detection task, leveraging the aforementioned automatic annotation and simply requiring free-access MI dialogues in the public domain without manually created empathy labels.

- We detail our proposed additional-input and multi-task detector extensions for explainability improvement.

\section{RELATED WORK}

The work we propose is closely related to prior work on 1) datadriven analysis of therapeutic empathy in MI and 2) text-based approaches to computational empathy in general conversation, as we will address the former using techniques inspired by the latter.

\subsection{Data-Driven Approaches to Therapeutic Empathy Analysis for MI}

Empathy is fundamental in coaching in general and in clinical counselling in particular, such as evidence-based behavioural treatments like MI. Prior work studying the link between empathy and MI delivery has focused on the speech and language of the therapist.

An early text-based attempt [35] adopted an n-gram language model for binary classification of utterance empathy. Gibson et al. [9] used generalised linear regression on other linguistic features as well as psycholinguistic norm features. More recently, long shortterm memory networks (LSTMs) [10] were used for turn-level encoding to infer utterance-level behaviours that are further used by a deep neural network (DNN) to predict session-level empathy [8].

The other line of work uses speech features. Xiao et al. [34] extracted prosodic features such as pitch and energy from speech signals for empathy classification using linear Support Vector Machines. Speech rate entrainment during dyadic interactions has also been investigated for its relationship to therapist empathy [38]. Finally, Pérez-Rosas et al. [19] trained a random forest on both linguistic and acoustic features to identify therapist empathy.

Note that previous work, as listed above, has all targeted offline therapist empathy assessment or identification of contributing factors to high-quality therapy. Our goal, in contrast, is to detect the need for therapist empathy, which makes our proposal closer to the work of Cao et al. [1], where a forecaster of MI behavioural codes was developed to provide real-time guidance for the therapist on what action (e.g. reflection/question/ $\cdots$ ) to take next. Our work differs from it in that we take an empathy-centred perspective.

It is worth mentioning that there is more recent work on deeplearning-powered MI analysis, including text-based [1, 7, 32, 36], speech-based [30], and multimodal [2] coding of therapist actions according to MI behaviour codes, but we do not consider this sphere of research since it is still predominantly for offline assessment of therapy quality and does not involve explicit empathy modelling.

\subsection{Text-Based Approaches to Computational Empathy in General Conversation}

Substantial progress has been achieved in text-based sentiment analysis (SA) [4, 24, 25, 27] in recent years, exemplified by the over$97 \%$ accuracies from the top entries in the SST-2 SA task on the 
GLUE [33] leaderboard ${ }^{4}$. In comparison, text-based empathy analysis, especially for general conversation, attracted less attention, until EMPATHETiCDiALOGUEs [23], a dataset of empathetic conversations grounded in emotions and situations, was made public. Since then, various studies have explored creating an empathetic opendomain conversation agent. Therefore, we focus on the approaches to computational empathy for such agents in this section.

Many of those agents incorporate current user emotion during their response generation. An early study [41] used emotion labels (emojis in tweets) as extra input to train a conditional variational encoder for response generation. Similarly, Lubis et al. [16] created an user-emotion context vector in addition to the HRED [31] response-generation framework. In [23], the emotion of the user is categorised as an emotion label to prepend each user utterance so that the response generator attends to user emotion explicitly. With GPT [21] as its backbone, CAiRE [14] adds an user-emotiondetection auxiliary objective in addition to the conventional response language modelling. Lin et al. [13], on the other hand, lined up specialised response generators that are each trained to reply to user utterances of a unique emotion.

Desirable or future emotion of the user or sentiment-aware agent has also been explored. Zandie and Mahoor [39] encouraged the agent to learn an appropriate emotion for its response, whereas Li et al. [12] conditioned their chatbot utterance on the desirable user emotion that the agent is trying to elicit. Within a reinforcement learning framework, Shin et al. [29] rewarded response candidates likely to induce positive user emotion.

\section{DATA}

The datasets we have considered for our problem are introduced below. Among them, $P E C$ will be used for the labelling step and HighRolePlayMI for the detecting step.

Persona-based Empathetic Conversation (PEC) [40] features $291 \mathrm{k}$ one-to-one general conversations crawled from 2 subreddits: $r /$ happy $^{5}$ and $r / o f f m y c h e s t^{6}$, both considered to consist mainly of empathetic conversations, as well as another $725 \mathrm{k}$ one-to-one casual conversations from $r$ /CasualConversation ${ }^{7}$ used as the control group, based on the assumption that casual conversations are less empathetic. The author verified the empathy and non-empathy of these conversation corpora with the "substantial agreement" shown by Fleiss' kappa [6] among the empathy annotators. We further group the $291 \mathrm{~K}$ empathetic conversations as EmpPEC and the $725 \mathrm{~K}$ non-empathetic ones as NonEmpPEC.

Pérez-Rosas et al. [20] created the first and, to the best of our knowledge, only publicly available dataset of MI conversations, which we term HighLowRolePlayMI. The dataset comprises 259 role-play conversations collected from public video-sharing sources such as YouTube and Vimeo, with 155 labelled as high-quality counselling and the other 104 as low-quality. The conversations are available as both videos and transcripts, without any extra annotation. We denote the high-quality counselling subset as HighRolePlayMI and the low-quality subset as LowRolePlayMI.

\footnotetext{
${ }^{4}$ https://gluebenchmark.com/leaderboard

${ }^{5} \mathrm{https}: / /$ www.reddit.com/r/happy/

${ }^{6} \mathrm{https} / /$ www.reddit.com/r/offmychest/

${ }^{7}$ https://www.reddit.com/r/CasualConversation
}

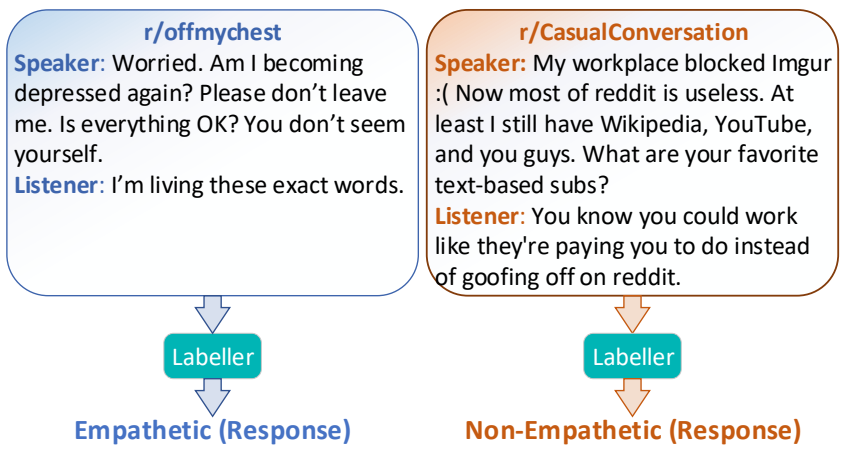

Figure 1: Training the labeller with a 2-utterance window over each $P E C$ conversation. Every listener reply in subreddits $r$ /happy and r/offmychest is considered an empathetic example, while every response in r/CasualConversation is deemed non-empathetic.

\section{PROPOSED METHODOLOGY}

Our method follows a labelling-detecting two-step setup. Both the labeller and the detector will have pre-trained language models as their underlying architectures. In particular, we will explore extending the detector with additional-input regimes and multi-task designs that can boost its performance and improve its explainability.

\subsection{Step 1: Labelling}

4.1.1 Task Definition. The labeller, $c s_{e m p}^{l a b e l}$, will be trained to determine whether a listener response is empathetic given the most recent speaker-listener talk turns. More concretely, cls sabp em will be given as input $H_{t, N}=\left\{u_{t-N+1}^{S}, u_{t-N+1}^{L}, \cdots, u_{t}^{S}, u_{t}^{L}\right\}$, a small window of the latest $N$ (e.g. $N=2$ ) exchanges between the interlocutors at time step $t$ where $u_{i}^{S}$ is the $i$-th speaker utterance and $u_{j}^{L}$ is the $j$-th listener utterance, and produce a binary classification on whether $u_{t}^{L}$ is an empathetic response to $u_{t}^{S}$. Note that we provide the $2 \mathrm{~N}$-utterance window to allow for more context for the labeller.

4.1.2 Training. Using PEC to train $c l s e m p$, we consider the initiator of a conversation as the speaker and the other interlocutor as the listener. Every listener response and its context in EmpPEC will be deemed a positive (empathetic) example, and likewise each listener reply with its context in NonEmpPEC will be used as a negative (non-empathetic) example, as shown in Figure 1.

We will use a pre-trained language model such as BERT [3] or GPT-2 [22] as the backbone of $c l s_{e m p}^{\text {label }}$. As input to BERT, for example, we will represent a 4-turn conversation, assuming a window length of 4 , as $\left\{[C L S],[S], u_{1}^{S},[L], u_{1}^{L},[S], u_{2}^{S},[L], u_{2}^{L}\right\}$, where $[S]$, $[L]$, and $[C L S]$ are special tokens. Specifically, $[S]$ indicates the utterance that follows is from the speaker, the same goes for $[L]$ for the listener, while the top-layer BERT representation of $[C L S]^{8}$ will be fed to a multi-layer perceptron (MLP) that will ultimately decide if $u_{2}^{L}$ is an empathetic response to $u_{2}^{S}$.

\footnotetext{
${ }^{8}[C L S]$ is a special token required by BERT [3] and its variants. It is placed at the beginning of the input, and its top-layer BERT representation is usually seen as a high-dimensional representation of the entire input. See [3] for more details.
} 


\section{HighRolePlayMI}

Client: I just have no energy, I don't wanna do anything but sleep Therapist: How long has that been going on? [Non-Empathetic]

Client: Well I've been drinking more. I mean I drink socially, but I find myself that, when I come home, you know, I have a glass of wine, and I just go to sleep.

Therapist: And that's worrisome to you. [Empathetic]

Client: It is, cuz I don't usually drink that often during the week. So, it's increased. Yeah.

\section{Client Needs Empathy Now? [YES]}

Figure 2: Training the detector with (partial) MI conversation history. The "non-empathetic" and "empathetic" labels of the therapist utterances are given by the labeller, with the client as the speaker and the therapist as the listener.

Once $c l s_{e m p}^{l a b e l}$ is trained, it will be used to annotate the MI corpus, considering the client as the speaker and the therapist as the listener, such that each therapist response in HighLowRolePlayMI is labelled as empathetic or non-empathetic.

\subsection{Step 2: Detection}

4.2.1 Task Definition. Once the accuracy for Step 1 has been validated, we define the need-for-empathy detection task as equivalent to determining whether the therapist should show empathy in their immediate response to the client, given the history of the conversations so far. More formally, we represent the therapeutic dialogue history as $H_{t}^{t h r}=\left\{u_{1}^{C}, u_{1}^{T}, u_{2}^{C}, u_{2}^{T}, \cdots, u_{t-1}^{C}, u_{t-1}^{T}, u_{t}^{C}\right\}$, where $u_{i}^{C}$ is the $i$-th client utterance and $u_{j}^{T}$ is the $j$-th therapist utterance, and the task is to decide whether $u_{t}^{T}$, the response to $u_{t}^{C}$, should be empathetic given $H_{t}^{t h r}$, as illustrated in Figure 2.

4.2.2 Baseline. We will train a baseline detector $c l s e m p$ on the nowannotated HighRolePlayMI to approach the task, with the input adapted from the representation described in Section 4.1.2. More formally, we will allow a much larger window of length $2 M+1(M \gg$ $N)$ for the most recent $2 M+1$ utterances: $H_{t, M}^{t h r}=\left\{u_{t-M}^{C}, u_{t-M}^{T}\right.$, $\left.\cdots, u_{t-1}^{C}, u_{t-1}^{T}, u_{t}^{C}\right\}$, and the input to the base pretrained language model, in the case of BERT [3] or its variants (e.g. ALBERT [11], RoBERTa [15]), will be $\left\{[C L S],[C], u_{t-M}^{C},[T], u_{t-M}^{T}, \cdots,[C], u_{t-1}^{C}\right.$, $\left.[T], u_{t-1}^{T},[C], u_{t}^{C}\right\}$, where $[C]$ is the special token for the client and $[T]$ for the therapist. The MLP above the language model will then reach a binary classification using the representation of the $[C L S]$, on whether $u_{t}^{T}$ needs to be empathetic.

Considering the potential susceptibility of this system to overfitting due to the small size of HighRolePlayMI, we propose 2 extensions to the baseline: emotion recognition and therapist response generation, as auxiliary objectives or for additional input. Their details are explained in the remainder of this section.

4.2.3 Extension: Emotion Recognition. Acknowledging emotions is essential to empathetic communication [23]. Considering that emotion recognition [26] is a well-resourced task, we hypothesise that involving emotions explicitly will be beneficial for the learning process of the detector and may enable more explainability.

For additional input to the baseline, an external emotion classifier $c s_{\text {emt }}$ will be trained on an emotion-labelled conversation dataset, e.g. EmpatheticDialogues [23], and provide an emotion label prepended at the beginning of each utterance before the conversation window is fed to $c l s_{e m p}^{d c t}$ as the input, similar to [23].

In a multi-task setting, we will feed the representation of [CLS] to two MLPs: one for need-for-empathy detection as in the baseline, and the other for emotion recognition. By assigning more weight to the detection loss and less to the recognition loss, we will achieve main (detection) - auxiliary (recognition) multi-task training.

4.2.4 Extension: Therapist Response Generation. $c l s s_{e m p}^{d c t}$ is effectively asked to forecast the empathy label of a therapist response absent in the input, which makes the task harder than the empathy labelling task. To facilitate the learning of the detector, we will leverage the actual therapist responses in HighRolePlayMI.

For additional input to the baseline, an external open-domain chatbot (e.g. [28]) will be fine-tuned on some counselling (not necessarily MI) conversation datasets to adapt to the therapeutic domain, and then used to, for each input, produce response candidates that are appended to the input in the need-for-empathy detection task.

In a multi-task setting, we can utilise the encoder-decoder backbone of an open-domain chatbot, but the encoder output is now fed to both 1) an MLP for need-for-empathy detection, and 2) the decoder for response generation. We make the detection the main task and the response generation the auxiliary task in a joint end-to-end training regime, which may allow for insights into the correlation between the detected need for empathy and the generated response.

\section{CONCLUSION}

We proposed a novel task of detecting client need for empathetic therapist response. Our plan for tackling this challenge is centred around a labeller-detector design, where we 1) first train a labeller on empathy-related general conversations so that it can automatically annotate an unlabelled MI corpus with binary empathy labels, and 2) then train a detector on the newly labelled MI corpus to detect real-time client need for empathetic response. We also laid out our plans for extending the detector with additional-input and multitask schemes to improve its ability and explainability. Expecting likely challenges in our experiments arising from the domain shift from general conversation to therapy, we hope our proposal and upcoming results will stimulate more research in empathy-related analysis for clinical counselling.

\section{ACKNOWLEDGMENTS}

This work has been funded by the EC in the H2020 Marie SkłodowskaCurie PhilHumans project, contract no. 812882. The authors would also like to thank Dr. Mark Aloia for his guidance and support.

\section{REFERENCES}

[1] Jie Cao, Michael Tanana, Zac E. Imel, Eric Poitras, David C. Atkins, and Vivek Srikumar. 2019. Observing Dialogue in Therapy: Categorizing and Forecasting Behavioral Codes. In Proceedings of the 57th Conference of the Association for Computational Linguistics, ACL 2019, Florence, Italy, July 28- August 2, 2019, Volume 1: Long Papers, Anna Korhonen, David R. Traum, and Lluís Màrquez 
(Eds.). Association for Computational Linguistics, 5599-5611. https://doi.org/10 18653/v1/p19-1563

[2] Zhuohao Chen, Karan Singla, James Gibson, Dogan Can, Zac E. Imel, David C Atkins, Panayiotis G. Georgiou, and Shrikanth S. Narayanan. 2019. Improving the Prediction of Therapist Behaviors in Addiction Counseling by Exploiting Class Confusions. In IEEE International Conference on Acoustics, Speech and Signal Processing, ICASSP 2019, Brighton, United Kingdom, May 12-17, 2019. IEEE, 66056609. https://doi.org/10.1109/ICASSP.2019.8682885

[3] Jacob Devlin, Ming-Wei Chang, Kenton Lee, and Kristina Toutanova. 2019. BERT Pre-training of Deep Bidirectional Transformers for Language Understanding. In Proceedings of the 2019 Conference of the North American Chapter of the Association for Computational Linguistics: Human Language Technologies, NAACL-HLT 2019, Minneapolis, MN, USA, June 2-7, 2019, Volume 1 (Long and Short Papers), Jill Burstein, Christy Doran, and Thamar Solorio (Eds.). Association for Computational Linguistics, 4171-4186. https://doi.org/10.18653/v1/n19-1423

[4] A. Dridi and D. Reforgiato Recupero. 2019. Leveraging semantics for sentiment polarity detection in social media. International fournal of Machine Learning and Cybernetics 10, 8 (2019), 2045-2055. https://doi.org/10.1007/s13042-017-0727-z cited By 17 .

[5] Robert Elliott, Arthur C Bohart, Jeanne C Watson, and Leslie S Greenberg. 2011. Empathy. Psychotherapy 48, 1 (2011), 43.

[6] Joseph L Fleiss. 1971. Measuring nominal scale agreement among many raters. Psychological bulletin 76, 5 (1971), 378.

[7] James Gibson, David C. Atkins, Torrey Creed, Zac E. Imel, Panayiotis G. Georgiou, and Shrikanth S. Narayanan. 2018. Multi-label Multi-task Deep Learning for Behavioral Coding. CoRR abs/1810.12349 (2018). arXiv:1810.12349 http://arxiv. org/abs/1810.12349

[8] James Gibson, Dogan Can, Bo Xiao, Zac E. Imel, David C. Atkins, Panayiotis G. Georgiou, and Shrikanth S. Narayanan. 2016. A Deep Learning Approach to Modeling Empathy in Addiction Counseling. In Interspeech 2016, 17th Annual Conference of the International Speech Communication Association, San Francisco, CA, USA, September 8-12, 2016, Nelson Morgan (Ed.). ISCA, 1447-1451. https: //doi.org/10.21437/Interspeech.2016-554

[9] James Gibson, Nikolaos Malandrakis, Francisco Romero, David C. Atkins, and Shrikanth S. Narayanan. 2015. Predicting therapist empathy in motivational interviews using language features inspired by psycholinguistic norms. In $I N$ TERSPEECH 2015, 16th Annual Conference of the International Speech Communication Association, Dresden, Germany, September 6-10, 2015. ISCA, 1947-1951. http://www.isca-speech.org/archive/interspeech_2015/i15_1947.html

[10] Sepp Hochreiter and Jürgen Schmidhuber. 1997. Long short-term memory. Neural computation 9, 8 (1997), 1735-1780.

[11] Zhenzhong Lan, Mingda Chen, Sebastian Goodman, Kevin Gimpel, Piyush Sharma, and Radu Soricut. 2020. ALBERT: A Lite BERT for Self-supervised Learning of Language Representations. In 8th International Conference on Learning Representations, ICLR 2020, Addis Ababa, Ethiopia, April 26-30, 2020. OpenReview.net. https://openreview.net/forum?id=H1eA7AEtvS

[12] Shifeng Li, Shi Feng, Daling Wang, Kaisong Song, Yifei Zhang, and Weichao Wang. 2020. EmoElicitor: An Open Domain Response Generation Model with User Emotional Reaction Awareness. In Proceedings of the Twenty-Ninth International Joint Conference on Artificial Intelligence, IfCAI 2020, Christian Bessiere (Ed.) ijcai.org, 3637-3643. https://doi.org/10.24963/ijcai.2020/503

[13] Zhaojiang Lin, Andrea Madotto, Jamin Shin, Peng Xu, and Pascale Fung. 2019. MoEL: Mixture of Empathetic Listeners. In Proceedings of the 2019 Conference on Empirical Methods in Natural Language Processing and the 9th International foint Conference on Natural Language Processing, EMNLP-IFCNLP 2019, Hong Kong, China, November 3-7, 2019, Kentaro Inui, Jing Jiang, Vincent Ng, and Xiaojun Wan (Eds.). Association for Computational Linguistics, 121-132. https://doi.org/ 10.18653/v1/D19-1012

[14] Zhaojiang Lin, Peng Xu, Genta Indra Winata, Farhad Bin Siddique, Zihan Liu, Jamin Shin, and Pascale Fung. 2020. CAiRE: An End-to-End Empathetic Chatbot. In The Thirty-Fourth AAAI Conference on Artificial Intelligence, AAAI 2020, The Thirty-Second Innovative Applications of Artificial Intelligence Conference, IAAI 2020, The Tenth AAAI Symposium on Educational Advances in Artificial Intelligence, EAAI 2020, New York, NY, USA, February 7-12, 2020. AAAI Press, 13622-13623. https://aaai.org/ojs/index.php/AAAI/article/view/7098

[15] Yinhan Liu, Myle Ott, Naman Goyal, Jingfei Du, Mandar Joshi, Danqi Chen, Omer Levy, Mike Lewis, Luke Zettlemoyer, and Veselin Stoyanov. 2019. RoBERTa: A Robustly Optimized BERT Pretraining Approach. CoRR abs/1907.11692 (2019) arXiv:1907.11692 http://arxiv.org/abs/1907.11692

[16] Nurul Lubis, Sakriani Sakti, Koichiro Yoshino, and Satoshi Nakamura. 2018. Eliciting Positive Emotion through Affect-Sensitive Dialogue Response Generation: A Neural Network Approach. In Proceedings of the Thirty-Second AAAI Conference on Artificial Intelligence, (AAAI-18), the 30th innovative Applications of Artificial Intelligence (IAAI-18), and the 8th AAAI Symposium on Educational Advances in Artificial Intelligence (EAAI-18), New Orleans, Louisiana, USA, February 2-7, 2018, Sheila A. McIlraith and Kilian Q. Weinberger (Eds.). AAAI Press, 5293-5300. https://www.aaai.org/ocs/index.php/AAAI/AAAI18/paper/view/16317
[17] William R Miller and Stephen Rollnick. 2012. Motivational interviewing: Helping people change. Guilford press.

[18] Theresa B Moyers, Lauren N Rowell, Jennifer K Manuel, Denise Ernst, and Jon M Houck. 2016. The motivational interviewing treatment integrity code (MITI 4): rationale, preliminary reliability and validity. Journal of substance abuse treatment 65 (2016), 36-42.

[19] Verónica Pérez-Rosas, Rada Mihalcea, Kenneth Resnicow, Satinder Singh, and Lawrence An. 2017. Understanding and Predicting Empathic Behavior in Counseling Therapy. In Proceedings of the 55th Annual Meeting of the Association for Computational Linguistics, ACL 2017, Vancouver, Canada, Fuly 30 - August 4, Volume 1: Long Papers, Regina Barzilay and Min-Yen Kan (Eds.). Association for Computational Linguistics, 1426-1435. https://doi.org/10.18653/v1/P17-1131

[20] Verónica Pérez-Rosas, Xinyi Wu, Kenneth Resnicow, and Rada Mihalcea. 2019. What Makes a Good Counselor? Learning to Distinguish between High-quality and Low-quality Counseling Conversations. In Proceedings of the 57th Annual Meeting of the Association for Computational Linguistics. Association for Computational Linguistics, Florence, Italy, 926-935. https://doi.org/10.18653/v1/P19-1088

[21] Alec Radford, Karthik Narasimhan, Tim Salimans, and Ilya Sutskever. 2018. Improving language understanding by generative pre-training (2018). URL https://s3-us-west-2. amazonaws. com/openai-assets/research-covers/languageunsupervised/language_understanding_paper. pdf (2018).

[22] Alec Radford, Jeffrey Wu, Rewon Child, David Luan, Dario Amodei, and Ilya Sutskever. 2019. Language models are unsupervised multitask learners. OpenAI Blog 1, 8 (2019), 9.

[23] Hannah Rashkin, Eric Michael Smith, Margaret Li, and Y-Lan Boureau. 2019. Towards Empathetic Open-domain Conversation Models: A New Benchmark and Dataset. In Proceedings of the 57th Conference of the Association for Computational Linguistics, ACL 2019, Florence, Italy, fuly 28-August 2, 2019, Volume 1: Long Papers, Anna Korhonen, David R. Traum, and Lluís Màrquez (Eds.). Association for Computational Linguistics, 5370-5381. https://doi.org/10.18653/v1/p19-1534

[24] D.R. Recupero, S. Consoli, A. Gangemi, A.G. Nuzzolese, and D. Spampinato. 2014. A semantic web based core engine to efficiently perform sentiment analysis. Lecture Notes in Computer Science (including subseries Lecture Notes in Artificial Intelligence and Lecture Notes in Bioinformatics) 8798 (2014), 245-248. https: //doi.org/10.1007/978-3-319-11955-7_28 cited By 17.

[25] D.R. Recupero, M. Dragoni, and V. Presutti. 2015. ESWC 15 challenge on conceptlevel sentiment analysis. Communications in Computer and Information Science 548 (2015), 211-222. https://doi.org/10.1007/978-3-319-25518-7_18 cited By 17.

[26] Diego Reforgiato Recupero, Mehwish Alam, Davide Buscaldi, Aude Grezka, and Farideh Tavazoee. 2019. Frame-Based Detection of Figurative Language in Tweets [Application Notes]. IEEE Comput. Intell. Mag. 14, 4 (2019), 77-88. https://doi. org/10.1109/MCI.2019.2937614

[27] D. Reforgiato Recupero and E. Cambria. 2014. ESWC'14 challenge on ConceptLevel Sentiment Analysis. Communications in Computer and Information Science 475 (2014), 3-20. https://doi.org/10.1007/978-3-319-12024-9_1 cited By 17.

[28] Stephen Roller, Emily Dinan, Naman Goyal, Da Ju, Mary Williamson, Yinhan Liu, Jing Xu, Myle Ott, Kurt Shuster, Eric Michael Smith, Y-Lan Boureau, and Jason Weston. 2020. Recipes for building an open-domain chatbot. CoRR abs/2004.13637 (2020). arXiv:2004.13637 https://arxiv.org/abs/2004.13637

[29] Jamin Shin, Peng Xu, Andrea Madotto, and Pascale Fung. 2020. Generating Empathetic Responses by Looking Ahead the User's Sentiment. In 2020 IEEE International Conference on Acoustics, Speech and Signal Processing, ICASSP 2020, Barcelona, Spain, May 4-8, 2020. IEEE, 7989-7993. https://doi.org/10.1109/ ICASSP40776.2020.9054379

[30] Karan Singla, Zhuohao Chen, David C. Atkins, and Shrikanth Narayanan. 2020. Towards end-2-end learning for predicting behavior codes from spoken utterances in psychotherapy conversations. In Proceedings of the 58th Annual Meeting of the Association for Computational Linguistics, ACL 2020, Online, fuly 5-10, 2020, Dan Jurafsky, Joyce Chai, Natalie Schluter, and Joel R. Tetreault (Eds.). Association for Computational Linguistics, 3797-3803. https://www.aclweb.org/anthology/ 2020.acl-main.351/

[31] Alessandro Sordoni, Yoshua Bengio, Hossein Vahabi, Christina Lioma, Jakob Grue Simonsen, and Jian-Yun Nie. 2015. A Hierarchical Recurrent Encoder-Decoder for Generative Context-Aware Query Suggestion. In Proceedings of the 24th ACM International Conference on Information and Knowledge Management, CIKM 2015, Melbourne, VIC, Australia, October 19 - 23, 2015, James Bailey, Alistair Moffat, Charu C. Aggarwal, Maarten de Rijke, Ravi Kumar, Vanessa Murdock, Timos K. Sellis, and Jeffrey Xu Yu (Eds.). ACM, 553-562. https://doi.org/10.1145/2806416. 2806493

[32] Michael Tanana, Kevin A Hallgren, Zac E Imel, David C Atkins, and Vivek Srikumar. 2016. A comparison of natural language processing methods for automated coding of motivational interviewing. Fournal of substance abuse treatment 65 (2016), 43-50.

[33] Alex Wang, Amanpreet Singh, Julian Michael, Felix Hill, Omer Levy, and Samuel R. Bowman. 2019. GLUE: A Multi-Task Benchmark and Analysis Platform for Natural Language Understanding. In 7th International Conference on Learning Representations, ICLR 2019, New Orleans, LA, USA, May 6-9, 2019. OpenReview.net. https://openreview.net/forum?id=rJ4km2R5t7 
[34] Bo Xiao, Daniel Bone, Maarten Van Segbroeck, Zac E. Imel, David C. Atkins, Panayiotis G. Georgiou, and Shrikanth S. Narayanan. 2014. Modeling therapist empathy through prosody in drug addiction counseling. In INTERSPEECH 2014 15th Annual Conference of the International Speech Communication Association, Singapore, September 14-18, 2014, Haizhou Li, Helen M. Meng, Bin Ma, Engsiong Chng, and Lei Xie (Eds.). ISCA, 213-217. http://www.isca-speech.org/archive/ interspeech_2014/i14_0213.html

[35] Bo Xiao, Dogan Can, Panayiotis G. Georgiou, David C. Atkins, and Shrikanth S Narayanan. 2012. Analyzing the language of therapist empathy in Motivational Interview based psychotherapy. In Asia-Pacific Signal and Information Processing Association Annual Summit and Conference, APSIPA 2012, Hollywood, CA, USA December 3-6, 2012. IEEE, 1-4. http://ieeexplore.ieee.org/document/6411762/

[36] Bo Xiao, Dogan Can, James Gibson, Zac E. Imel, David C. Atkins, Panayiotis G Georgiou, and Shrikanth S. Narayanan. 2016. Behavioral Coding of Therapist Language in Addiction Counseling Using Recurrent Neural Networks. In Interspeech 2016, 17th Annual Conference of the International Speech Communication Association, San Francisco, CA, USA, September 8-12, 2016, Nelson Morgan (Ed.) ISCA, 908-912. https://doi.org/10.21437/Interspeech.2016-1560

[37] Bo Xiao, Che-Wei Huang, Zac E. Imel, David C. Atkins, Panayiotis G. Georgiou, and Shrikanth S. Narayanan. 2016. A technology prototype system for rating therapist empathy from audio recordings in addiction counseling. Peerf Comput.
Sci. 2 (2016), e59. https://doi.org/10.7717/peerj-cs.59

[38] Bo Xiao, Zac E. Imel, David C. Atkins, Panayiotis G. Georgiou, and Shrikanth S. Narayanan. 2015. Analyzing speech rate entrainment and its relation to therapist empathy in drug addiction counseling. In INTERSPEECH 2015, 16th Annual Conference of the International Speech Communication Association, Dresden, Germany, September 6-10, 2015. ISCA, 2489-2493. http://www.isca-speech.org/archive/ interspeech_2015/i15_2489.html

[39] Rohola Zandie and Mohammad H. Mahoor. 2020. EmpTransfo: A Multi-Head Transformer Architecture for Creating Empathetic Dialog Systems. In Proceedings of the Thirty-Third International Florida Artificial Intelligence Research Society Conference, Originally to be held in North Miami Beach, Florida, USA, May 17-20, 2020, Roman Barták and Eric Bell (Eds.). AAAI Press, 276-281. https://aaai.org/ ocs/index.php/FLAIRS/FLAIRS20/paper/view/18446

[40] Peixiang Zhong, Yan Sun, Yong Liu, Chen Zhang, Hao Wang, Zaiqing Nie, and Chunyan Miao. 2020. Endowing Empathetic Dialogue Systems with Personas. CoRR abs/2004.12316 (2020). arXiv:2004.12316 https://arxiv.org/abs/2004.12316

[41] Xianda Zhou and William Yang Wang. 2018. MojiTalk: Generating Emotional Responses at Scale. In Proceedings of the 56th Annual Meeting of the Association for Computational Linguistics, ACL 2018, Melbourne, Australia, July 15-20, 2018, Volume 1: Long Papers, Iryna Gurevych and Yusuke Miyao (Eds.). Association for Computational Linguistics, 1128-1137. https://doi.org/10.18653/v1/P18-1104 